\title{
OVERVIEW OF EDGE COMPUTING IN SMALL BUSINESSES
}

\author{
Jessica Schwartz, University of the Cumberlands, jessica.schwartz@ucumberlands.edu \\ Loreen Powell, Bloomsburg University, Ipowell@bloomu.edu \\ Michalina Hendon, University of the Cumberlands, michalina.hendon@ucumberlands.edu
}

\begin{abstract}
Edge computing is regarded as an enabling computation and data storage technology that brings data closer to the location needed (i.e. closer to the network edge) to optimize Internet of Things (IoT) devices and web applications. With edge computing, one can expect faster response times and utilize less bandwidth. In response to COVID-19, small businesses are relying on edge computing to stay in business. The evolution of information and communication technologies (ICT) has advanced at an unprecedented rate, leading to the growth of many applications such as the IoT. As an important emerging technology, edge computing plays a critical role in different technological applications and helps to deliver a rich variety of services and data in real time for small businesses. This theoretical paper explores edge computing for small businesses and provides recommendations for small businesses to utilize edge computing successfully in the future, especially with the growth of $5 G$ networking.
\end{abstract}

Keywords: Edge Computing, Small Business, IoT, Data Transmission, Processing Techniques, 5G

\section{INTRODUCTION}

Exponential growth in technological advancements continues to be integrated into business operations, resulting in new innovations. One such innovation is edge computing, which has become the modern way of maximizing the usefulness of advanced technology and data processing. Organizations are increasingly realizing the importance of edge computing and rely on this innovation to improve data management practices (Guynes et al., 2020). As Munn (2020) defines, "the edge is both a paradigm and an architecture that aims to store and process data closer to the source" (p.270). Edge computing relates to a distributed computing topology of a given network where information processing is converged at the edge, which are typically its access points. The edge of the access point is the point at which individuals and organizations can readily access information for consumption (Guynes et al., 2020).

Small businesses can benefit from the adoption of edge computing as the businesses seek efficiency by utilizing edge computing data management processes. Unlike cloud computing, edge computing allows a device to process data either internally through an edge device, a local server, or a local computer rather than using a data center. Edge computing has been of great help to organizations, especially small businesses, due to its efficiency in data transmission (Guynes et al., 2020). As an example, small healthcare businesses need to access essential patient data. Utilizing edge computing rather than traditional methods (such as fragmented databases), the business would have a faster and more efficient way to access needed data. Another example would be the use of small solar power. Businesses generate a massive amount of useful data that can provide analytics to utility companies. Businesses can then utilize edge computing to perform advanced real-time monitoring and analytics capabilities. This will assist in generating actionable and valuable insights on distributed resources (such as renewable energy).

Majority of edge computing literature focuses on the background of edge computing and what the potential is for this technology. However, there is a lack of resources focusing on edge computing for small businesses. The purpose of this paper is twofold. First, it explores the relevant body of knowledge on the adoption of edge computing for small businesses. Second, it identifies some of the reasons why edge computing for small businesses has not yet gained widespread adoption. The remainder of this paper is structured as follows: background/review of literature, techniques, recommendations, and conclusion. 


\section{BACKGROUND/REVIEW OF LITERATURE}

Typically, small businesses leverage information technology (IT) to augment operations and transaction processing activities along with supporting and fostering customer relationships (Ramaswamy, 2019). However, due to COVID19, almost every organization relies on IT to keep its business open. As a result, there is an increase in the number of small businesses harnessing edge computing. Edge computing can be integrated in businesses, so users can access data, analyze data, and utilize given data effectively. Edge computing is a technological solution, which has been derived from cloud/fog computing and the IoT.

Cloud computing gave rise to edge computing where data access was leveraged at the lowest level of access. Likewise, IoT is an integration of devices in a given network or computing topology, which share data in the form of smart systems (Rawat, 2019). Cloud computing and IoT can make individuals and organizations efficiently process and analyze data at the lowest edge possible, hence the concept of edge computing.

Extensive growth of IoT devices, which receive a vast amount of resources from the cloud, make edge computing possible. With edge computing, data can be retrieved from cloud computing and processed, and then be delivered back to the cloud when the given user no longer needs access to that data (Wang et al., 2018). In a way, edge computing makes data processing possible on mobile devices. Edge computing is closer to users than cloud computing, therefore leading to faster response times.

Small businesses could improve upon their business operations by utilizing edge computing. Most computing devices are becoming less expensive to acquire and integrate into core business operations. This shift could increase the affordability as well as the use and integration of the technology (Wang et al., 2018). Additionally, given the rapid growth of IoT and cloud computing, it is relatively easy for small businesses to maximize the availability of edge computing to increase their data storage and processing capabilities.

\section{Reduced Latency}

A small business needs to ensure their data is transmitted without latency to serve customers and internal business processes effectively and efficiency. Latency is the process where data is available, but there are delays during the transfer process (Taleb et al., 2017). With edge computing, information is stored in the edge of a network where it can be easier to access data. This process allows relocation of data and different computing tasks closer to the user, which results in faster responsive services.

Edge and cloud computing use 5G networks, which is fast and increases bandwidth. This will impact a small business operation where it will be possible to transmit massive volumes of data within a short period, and therefore be able to serve more customers. On data storage, the use of a 5G network allows organizations and individuals to store vast amounts of data (Mastorakis et al., 2020).

\section{Cost Efficiency}

Edge computing is less expensive to use, implement, and store data compared to cloud computing. One challenge faced by small businesses include lack of capital (Roman et al., 2018). Small businesses can use edge computing to ensure services are run effectively and quality service is provided to customers. Edge computing allows users to store data on their devices and computers, thereby eliminating the need for additional data servers stored offsite. Additionally, transmitting data from servers in different locations to the user requires a more robust network, which might be expensive for small businesses to acquire. The infrastructure of edge computing can be found to be more affordable for smaller businesses as edge computing may be as simple as running the infrastructure on an IoT device (Scale Computing, 2020). 


\section{Issues in Information Systems}

Volume 21, Issue 3, pp. 200-206, 2020

\section{Scalability}

With time, small businesses will expand and require the development of their storage system depending on the amount of data been transmitted in the system. The use of a centralized form of computing limits an organization in developing its storage system (Gyarmathy, 2019). Centralized computing involves the establishment of data centers that are expensive to construct, maintain, and expand or replace when there are new developments within the organization (Gyarmathy, 2019). With edge computing, it is possible to expand the systems in the future in a less expensive model. Decentralized computing is more flexible to changes and easily adaptable to new systems with scalable options.

\section{Increased Security}

Most small businesses are facing the challenges of cybercrimes, where data is among the main target of cybercriminals. Small businesses need to be fully prepared with mitigation tools which can aid in the security of their operations. With the use of edge computing, an organization can monitor all operations in the network. Secondly, all processes are distributed in a wide range of data centers and storage devices, which limits the chances of a single attack disrupting all the activities in the organization (Gyarmathy, 2019). Lastly, data is transmitted to the local devices; hence, the only data at risk is the locally collected data on a specific device.

\section{Wireless Networks}

Storage and computing power can be brought to the edge with wireless networks to offer real time computing power to users. According to Rawat (2019), edge computing plays a major role within wireless networks in areas such as smart energy grid and vehicular wireless networks. As an example, small businesses aiming to provide vehicular wireless networks can utilize edge computing to deliver a variety of services in real time for end users to enhance traffic efficiency and road safety. Wireless networks that take advantage of processing data at the edge are more efficient especially in processing large data sets (i.e. big data) (Rawat, 2019).

\section{EDGE COMPUTING TECHNIQUES}

Edge computing applications are still in development infancy (Bagchi et al., 2020). One approach for a small business to utilize edge computing is through massive data storage. However, data must first be integrated into edge computing before transferring the given data to the cloud. Data stored within the edge computing environment could be easily accessed, including through remote methods, and become useful to organizations (Wang et al., 2018). In comparison to large organizations, small businesses generate less data based on the scope of their operations. For quick retrieval and ease of storing, small businesses could use edge computing to store data much more efficiently than cloud computing or traditional storage methods.

Small businesses could further use edge computing to ensure faster data processing. Organizations are currently integrating 5G technologies, in which edge computing is embedded with this technology (Mastorakis et al., 2020). It is possible to use edge computing to ensure faster data processing, given that edge computing is characterized by high latency. High processing internet speeds, as further integrated within the edge computing, could provide efficient data processing for small businesses.

Most small businesses could utilize edge computing to ensure automation of critical business functions. Edge computing has started to become integrated with artificial intelligence and smart systems, where technological advancements are converging at making edge computing intelligent. Currently, many edge devices and edge computing interactions diminish network necessity, thus creating low latency information processing centered around client devices (Bagchi et al., 2020). Small businesses could use edge technology to automate some processes such as responding to customer inquiries and order handling, which will enable businesses to achieve efficiency and economy in daily business functions.

As data security continues to become a challenge to most organizations, small businesses could utilize edge computing to ensure the security of their data and applications. Edge computing is becoming distributed with advanced security enhancement mechanisms. Small businesses can incorporate edge computing as a way of ensuring benefit from 


\section{Issues in Information Systems}

Volume 21, Issue 3, pp. 200-206, 2020

advanced security measures (Mastorakis et al., 2020). For instance, tunneling and utilization of a virtual private network (VPN) are some of the critical security measures integrated into edge computing. Small businesses can utilize edge computing as a way of enhancing the essential security of organizational data.

Increased interconnectivity within the edge computing environment will enable small businesses to use this technology to derive customized solutions (Bagchi et al., 2020). As edge computing becomes integrated into the modern computing environment, advanced technologies such as operating systems, internet connectivity, and the use of smart devices, edge computing becomes ideal in addressing most of the modern-day business problems (Zhang et al., 2018). Small businesses can take advantage of edge computing to ensure that they provide customized products and services, which serve the need of their customers. It is important to note that edge computing integration seems reasonable when deployed on a small scale (Bagchi et al., 2020). However, several challenges are expected as the number of edge applications and data increase as IoT device usage increases.

As many organizations pay for cloud storage, small businesses can leverage data storage whether stored on the cloud or elsewhere. Edge computing can assist in data filtering by sending only useful information to the cloud. Data filtering undergoes processing to decrease unnecessary data, thus storing only necessary data (Li et al., 2018).

\section{Leveraging the Edge}

Several techniques are viable for small businesses in utilizing edge computing technological solutions, such as system design, adoption of advanced computing devices, security modeling, and protocol design (Zhang et al., 2018). One of the critical characteristics of edge computing is heterogeneity in the computing devices used. Increased heterogeneity could be a challenging idea to small businesses in effectively utilizing edge computing, hence the need to adopt advanced computing techniques (Zhang et al., 2018). Security modeling, protocol design, and other vital advancements could provide viable techniques to small businesses in ensuring maximum advantage of edge computing.

The integration of advanced edge computing techniques could further provide innovative ways in which small businesses can take advantage of edge computing. Data aggregation techniques, points of interest, crowdsourcing, and traffic management across the edge computing environment are ideal edge computing techniques for small businesses (Zhang et al., 2018). Although edge computing is becoming more widely adopted, there are critical security threats, which could compromise edge computing benefits. Therefore, the integration of advanced computing techniques could ensure that small businesses benefit from this innovative technology.

To keep pace with larger businesses, a competitive advantage for small businesses involves utilizing edge computing to track trends, buying behaviors, and market products to retail shoppers with the use of smartphone applications that can be used in a brick and mortar store. Using edge computing, business can begin to collect data on customer behaviors and decision-making processes (Premsankar et al., 2018). With the use of the data predictive models, the future behavior of customers can be established and used to enhance overall customer experience (Scale Computing, 2020). Edge computing can also be used to synchronize with the customer using mobile devices to enhance the customer experience by offering customized mobile coupons that will populate when the application is used while in the range of the store's location.

The benefits of using edge computing for small business include turnkey solutions that lessens the need for an experienced technology director on staff. As most of edge computing runs from a hub or edge device, they are preprogrammed to work out of the box. The data trafficking built-in will assist with directing the usable to siphon to a central depository of data which then can be archived to the cloud (Sittón-Candanedo et al., 2019).

\section{Limitations to Edge Computing}

There are limitations that are integral to a $4 \mathrm{G}$ network. In the current $4 \mathrm{G}$ technology environment, there are different bandwidths, latency, and density requirements which depend upon separate network physicality to transfer the data. The technology that 5G can offer includes faster applications and device data processing with the use of edge 
computing system devices. With the use of a 5G network, data can be funneled into the edge devices where only pertinent information is gathered, stored, and archived to cloud storage in a real-time data transfer (Pham et al., 2019).

As the United States begins the shift to 5G, it should be noted that the infrastructure of 5G architecture is being deployed in geographic phases and will add to the established core data centers, metro-level edge clouds, as well as far edge clouds according to FCC Technological Advisory Council (n.d). As the architecture and devices are upgrading to $5 \mathrm{G}$ on top of $4 \mathrm{G}$ technology, anticipation of only $74 \%$ of mobile subscriptions will be upgraded to $5 \mathrm{G}$ by the end of 2025 (Ericsson, 2019). Once devices are more accessible to 5G, programs can be developed to take full advantage of the infrastructure using edge computing.

\section{RECOMMENDATIONS FOR FUTURE RESEARCH}

Although edge computing has advantages, such as minimizing costs and improving overall business efficiency, small businesses must take a proactive risk management approach in adopting this technology (Premsankar et al., 2018). By doing so, small businesses can minimize the risk of critical security challenges, which could compromise edge computing security. Notably, edge computing offers a large surface area of attack due to its distributed nature. Through a risk management approach, small businesses could potentially minimize the threats and security challenges that could compromise the functioning of edge computing. Suggested research questions that surround the use of edge computing for small businesses could include the question of cost advantage in both a perspective of customer use as well as the cost of maintenance and integration of the technology. Once edge computing is integrated in a more robust manner future research can begin to collect the data of how the use of the customer connections to the edge server provide a both a marketing advantage as well as gauging the cost savings.

Since edge computing facilitates high data processing and storage capabilities, small businesses can use big data analytics to improve their data management practices (Premsankar et al., 2018). One such practice is using real-time data analysis to improve the performance of small businesses. Additionally, augmented reality could be achieved through the incorporation of edge computing.

Edge computing has the capability to provide big data analytics for predicting network capacity for small businesses (Rawat, 2019). Future research could be conducted to identify the role of big data analytics with the use of edge computing to improve effectiveness for organizational decision making. An empirical analysis of both edge computing and big data analytic architectures can be identified in future research to determine the validation of effectiveness and performance of edge computing as it relates to big data analytics.

\section{CONCLUSION}

In conclusion, the use of edge computing can play a vital role in the growth of small businesses. A sample of the benefits of utilizing edge computing includes increased security and reduced latency to allow data is transmitted with higher speeds with the use of 5G networks. Edge computing enhances scalability, where the business can quickly adapt to new changes in the future. Lastly, edge computing ensures reliability. As a result, small businesses can transmit data and store data more securely and less expensively.

Edge computing is appealing and beneficial to small businesses, especially when the appropriate computing techniques are integrated into the edge computing environment. Notably, small businesses could utilize edge computing by taking advantage of security enhancements embedded within this technology to ensure more data storage and data processing. Lastly, small businesses may achieve higher business value by using edge computing to create customized products and services.

It is important to note that this paper is not without limitations. First, this research is limited in that it is theoretical in nature. Second, this study did not test any of the techniques described. Future research should address the above limitations. 
This paper has practical implications for small businesses, higher education institutions, and faculty. Implications include an overview of the state of the art and knowledge for practitioners and academics as a teaching resource regarding edge computing for small businesses.

\section{REFERENCES}

Bagchi, S., Siddiqui, M-B., Wood, P., \& Zhang, H. (2020). Dependability in edge computing. Communications of the ACM., 63(1), 58-66.

Ericsson. (2019). Ericsson Mobility Report. Retrieved from https://www.ericsson.com/4acd7e/assets/local/mobilityreport/documents/2019/emr-november-2019.pdf

FCC Technological Advisory Council. (n.d). 5G Edge Computing White Paper (White Paper). Federal Communications Commission.

Guynes, S., Parrish, J., \& Vedder, R. (2020). Edge computing societal privacy and security issues. ACM SIGCAS Computers and Society, 48(3-4), 11-12.

Gyarmathy, K. (2019). The benefits and potential of edge computing. vXchnge. Retrieved from https://www.vxchnge.com/blog/the-5-best-benefits-of-edge-computing

Li, M., Yu, F. R., Si, P., \& Zhang, Y. (2018). Green machine-to-machine communications with mobile edge computing and wireless network virtualization, IEEE Communications Magazine, 56(5), 148-154.

Mastorakis, S., Mtibaa, A., Lee, J., \& Misra, S. (2020). ICedge: When edge computing meets information-centric networking. IEEE Internet of Things Journal, 7(5), 4203-4217.

Mor, N. (2019). Research for practice: Edge computing. Communications of the ACM, 62(4), 95-99.

Munn, L. (2020). Staying at the Edge of Privacy: Edge Computing and Impersonal Extraction. Media and Communication, 8(2), 270-279. https://doi.org/10.17645/mac.v8i2.2761

Pham, Q. V., Fang, F., Ha, V. N., Le, M., Ding, Z., Le, L. B., \& Hwang, W. J. (2019). A survey of multi-access edge computing in 5G and beyond: Fundamentals, technology integration, and state-of-the-art. Symmetry, 11, 842859.

Premsankar, G., Di Francesco, M., \& Taleb, T. (2018). Edge computing for the Internet of Things: A case study. IEEE Internet of Things Journal, 5(2), 1275-1284.

Ramaswamy, M. (2019). Information technology strategies for small businesses. Issues in Information Systems, 20(2), 216-225. Retrieved from https://iacis.org/iis/2019/2_iis_2019_216-225.pdf

Rawat, D. B. (2019). Fusion of software defined networking, edge computing, and blockchain technology for wireless network virtualization. IEEE Communications Magazine, 50-55.

Roman, R., Lopez, J., \& Mambo, M. (2018). Mobile edge computing: A survey and analysis of security threats and challenges. Future Generation Computer Systems, 78, 680-698.

Scale Computing. (2020). Retail on the edge from the experts at scale computing. Scale Computing. Retrieved from https://www.scalecomputing.com/documents/Retail-On-The-Edge-White-Paper-Published-1-2020.pdf

Sittón-Candanedo, I., Alonso, R. S., García, Ó., Muñoz, L., \& Rodríguez-González, S. (2019). Edge computing, IoT and social computing in smart energy scenarios. Sensors (Basel, Switzerland), 19(15). Retrieved from https://doi-org.proxy-bloomu.klnpa.org/10.3390/s19153353 


\section{Issues in Information Systems}

Volume 21, Issue 3, pp. 200-206, 2020

Taleb, T., Samdanis, K., Mada, B., Flinck, H., Dutta, S., \& Sabella, D. (2017). On multi-access edge computing: A survey of the emerging 5G network edge cloud architecture and orchestration. IEEE Communications Surveys \& Tutorials, 19(3), 1657-1681.

Wang, T., Zhang, G., Liu, A., Bhuiyan, M. Z. A., \& Jin, Q. (2018). A secure IoT service architecture with an efficient balance dynamics based on cloud and edge computing. IEEE Internet of Things Journal, 6(3), 4831-4843.

Zhang, K., Leng, S., He, Y., Maharjan, S., \& Zhang, Y. (2018). Cooperative content caching in 5G networks with mobile edge computing. IEEE Wireless Communications, 25(3), 80-87. 